\title{
Initial Investigations of Controller Tools and Procedures for Schedule-Based Arrival Operations with Mixed Flight-Deck Interval Management Equipage
}

\author{
Todd J. Callantine, ${ }^{1}$ Christopher D. Cabrall, ${ }^{2}$ Michael Kupfer, ${ }^{3}$ and Faisal G. $\mathrm{Omar}^{4}$ \\ San Jose State University/NASA Ames Research Center, Moffett Field, CA, 94066 \\ Thomas Prevot ${ }^{5}$ \\ NASA Ames Research Center, Moffett Field, CA, 94066
}

\begin{abstract}
NASA's Air Traffic Management Demonstration-1 (ATD-1) is a multi-year effort to demonstrate high-throughput, fuel-efficient arrivals at a major U.S. airport using NASAdeveloped scheduling automation, controller decision-support tools, and ADS-B-enabled Flight-Deck Interval Management (FIM) avionics. First-year accomplishments include the development of a concept of operations for managing scheduled arrivals flying Optimized Profile Descents with equipped aircraft conducting FIM operations, and the integration of laboratory prototypes of the core ATD-1 technologies. Following each integration phase, a human-in-the-loop simulation was conducted to evaluate and refine controller tools, procedures, and clearance phraseology. From a ground-side perspective, the results indicate the concept is viable and the operations are safe and acceptable. Additional training is required for smooth operations that yield notable benefits, particularly in the areas of FIM operations and clearance phraseology.
\end{abstract}

\section{Introduction}

$\mathrm{T}$ RAJECTORY-BASED traffic management tools, precision arrivals, improved surveillance, and precise interarrival spacing are key elements of the FAA's NextGen Implementation Plan for improving efficiency and capacity at busy airports. ${ }^{1}$ The plan emphasizes Performance-Based Navigation (PBN) approaches to streamlining arrival management operations to save fuel, reduce noise, and maximize the use of existing capacity. Among these are scheduling arrivals to fly deconflicted Optimized Profile Descents (OPDs), and using Automatic Dependent Surveillance-Broadcast (ADS-B) to enable Flight-Deck Interval Management (FIM) for increased inter-arrival precision. NASA recently inaugurated Air Traffic Management Demonstration-1 (ATD-1) to demonstrate these capabilities using NASA-developed scheduling automation, controller decision-support tools, and ADS-B-enabled FIM avionics. ATD-1 is conceived as a multi-year collaborative effort between researchers at NASA Ames and Langley Research Centers, the FAA, and industry partners to integrate and mature the technologies to the level necessary for operational demonstration, collect operational data on fuel-savings benefits and the acceptability of operations by participating flight crews and controllers, and provide these data to partners and stakeholders to support infrastructure and avionics investment decisions. ${ }^{2}$

The ATD-1 integrated arrival solution (Fig. 1) is called the Interval Management Terminal-Area Precision Scheduling System (IM-TAPSS). ${ }^{3}$ The scheduling component of IM-TAPSS is the Traffic Management Advisor for Terminal Metering (TMA-TM) developed at NASA Ames. TMA-TM is an extension to the currently deployed TMA automation system that uses trajectory predictions along terminal-area routes to construct arrival schedules tailored for high-throughput OPD operations. ${ }^{4}$ Controller-Managed Spacing (CMS) tools developed in the Airspace

${ }^{1}$ Senior Research Engineer, Human Systems Integration Division, NASA Ames Mail Stop 262-4, AIAA Senior Member.

${ }^{2}$ Senior Research Psychologist, Human Systems Integration Division, NASA Ames Mail Stop 262-4.

${ }^{3}$ Senior Research Engineer, Human Systems Integration Division, NASA Ames Mail Stop 262-4, AIAA Member.

${ }^{4}$ Research Associate, Human Systems Integration Division, NASA Ames Mail Stop 262-4.

${ }^{5}$ Research Engineer, Human Systems Integration Division, NASA Ames Mail Stop 262-4, AIAA Senior Member. 


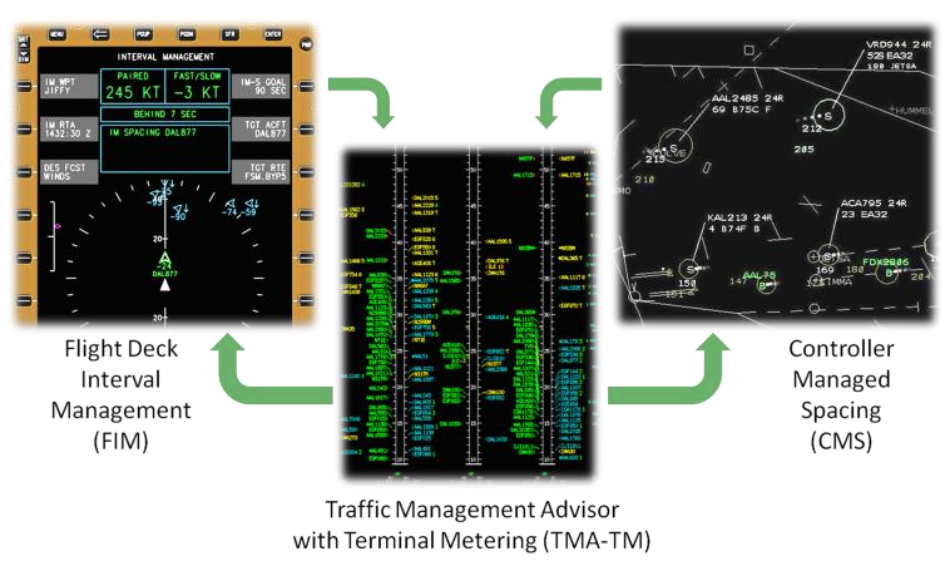

Figure 1. IM-TAPPS core technologies.

Operations Laboratory (AOL) at NASA Ames enable controllers to maximize runway throughput by continuing to manage aircraft toward TMA-TM schedules in the terminal area. CMS tools also aid controllers in using speed control to meet the schedules, thereby reducing the need for tactical vectoring and limiting OPD interruptions. ${ }^{5}$ FIM capabilities in IMTAPSS are implemented using the Airborne Spacing for Terminal Arrival Routes (ASTAR) algorithm developed at NASA Langley Research Center. ${ }^{6}$ ASTAR uses ADS-B data to provide flight crews speed commands along published OPDs, enabling aircraft to precisely achieve an assigned spacing interval at a specified 'achieve-by'
r workload and contribute additional interpoint. FIM operations are expected to reduce terminal-area controller workload and contribute additi
arrival precision for increasing the proportion of uninterrupted OPDs achievable with high throughput.

Important accomplishments during the first year of ATD-1 include initial partnership development with the FAA and other stakeholders, feasibility studies for integrating the CMS tools with operational terminal-area controller displays, and the development of a concept of operations (ConOps) that specifies procedures, phraseology, and example scenarios for managing mixed-FIM-equipage arrivals with IM-TAPSS. ${ }^{7,8}$ In parallel with these efforts, collaborative laboratory-integration activities were conducted to install the core IM-TAPSS components in air traffic laboratories at NASA Ames and Langley Research Centers. The AOL served as the systems-integration testbed, where a laboratory-prototype TMA-TM and ASTAR-equipped Aircraft Simulator for Traffic Operations Research (ASTOR) simulators from NASA Langley were initially integrated with the AOL's Multi-Aircraft Control System (MACS) controller workstation emulations and pseudo-pilot stations. ${ }^{9}$ After each phase of integration work, updated simulation components were migrated between the various ATD-1 laboratories.

A human-in-the-loop simulation was also conducted in the AOL following each integration phase. The first simulation (CMS ATD-1 \#1, or 'CA-1') in January 2012 provided an initial look at mixed-FIM-equipage operations, with controllers using the CMS tools to manage unequipped aircraft while ASTOR pilots conducted FIM operations. It was followed by CA-2 in April 2012, and CA-3 in June 2012. Each week-long simulation afforded the opportunity to collect data using the latest IM-TAPSS laboratory prototype, gather feedback from pilot and controller participants, and refine the controller tools, procedures, and phraseology. This paper presents these simulations from a primarily ground-side perspective, with an emphasis on the most recent CA-3 simulation. Section II provides an overview of the ATD-1 ConOps, including general descriptions of the procedures, clearance phraseology, and controller decision-support tools. Following brief descriptions of the CA-1 and CA-2 studies, Section III describes the CA-3 experiment and results in detail. The paper concludes with a discussion of key findings relevant for ConOps refinements and future ATD-1 development efforts.

\section{ATD-1 Concept of Operations}

Integrated scheduling and spacing operations supported by IM-TAPSS are intended to provide efficiency benefits at airports served by OPDs when traffic levels would not otherwise permit uninterrupted OPDs. OPDs are typically published Area Navigation (RNAV) routes with speed and altitude restrictions that include runway transitions connected to instrument approach procedures, so that flight crews can use onboard Flight Management System (FMS) capabilities to fly them from cruise to landing. The published speed restrictions define a nominal speed profile; IM-TAPSS leverages the nominal speed profile for scheduling and provides mechanisms for adjusting it to achieve the arrival schedule while maintaining the lateral and vertical profiles. This section describes nominal ATD-1 operations using IM-TAPSS, introduces the CMS tools, and provides examples of clearance phraseology.

\section{A. Operations in Center Airspace}

Operations begin with aircraft in cruise navigating toward their top-of-descent points. The TMA-TM acquires each aircraft some site-specific distance (e.g., $200 \mathrm{nmi}$ ) from the Terminal Radar Approach Control (TRACON) boundary. The TMA-TM assigns the aircraft a runway, and computes an estimated time-of-arrival (ETA) at the 
meter fix, runway, and at intervening TRACON metering points (e.g., fixes where OPDs merge). TMA-TM then uses the ETAs to assign each aircraft a scheduled-time-of-arrival (STA) at each point. The TMA-TM uses sitespecific adaptation information about the OPDs in its trajectory predictions, and allocates delay required between each scheduling point such that speed control is sufficient to maintain aircraft separation. This delay-allocation scheme ensures aircraft absorb large delays at higher altitudes, which is typically more efficient.

As each aircraft reaches a prespecified 'freeze horizon,' the TMA-TM locks in its STA to provide a stable control target. En-route controllers now begin working to 'precondition' the aircraft using vectoring or other techniques as necessary to reduce the delay required to within the speed control margin. Ongoing FAA en-route modernization efforts are expected to include tools to aid controllers in preconditioning aircraft efficiently. Following preconditioning, controllers reestablish aircraft on RNAV routes (e.g., by clearing aircraft direct to the meter fix on the TRACON boundary), and clear aircraft to 'descend-via' their assigned OPDs. The clearance phraseology is expected to follow emerging FAA standards and include the TMA-TM runway assignment: <CALLSIGN>, DESCEND VIA THE MAIER THREE ARRIVAL, BOULDER CITY TRANSTION, TO RUNWAY TWO-SIX.

Once aircraft are preconditioned for speed control and established on an RNAV procedure, controllers issue FIM clearances to FIM-equipped aircraft. The ASTAR algorithm accepts several parameters that enable it to provide FIM guidance for achieving a desired spacing interval at a specific 'achieve-by' point behind an assigned lead, or 'target,' aircraft that may be flying a different arrival route. ASTAR can also command speeds toward a required time-ofarrival (RTA) at the achieve-by point until a target flying a different route enters ADS-B range. Providing the required parameters to ASTAR is a simple matter in data-communications environments ASTAR was originally developed to work in; ATD-1 is pursuing phraseology for issuing the FIM clearance via voice communications. The following clearance phraseology assumes the achieve-by point is the same as the RTA point (i.e., the Final Approach Fix (FAF) 'JIFFY'): <CALLSIGN>, FOR INTERVAL SPACING, CROSS JIFFY AT 1432:30Z. WHEN ABLE, SPACE NINE-ZERO SECONDS BEHIND DELTA EIGHT-SEVEN-SEVEN ON THE BONHAM FIVE ARRIVAL, FORT SMITH TRANSITION. In IM-TAPSS, the target and spacing interval are determined from the TMA-TM schedule at the achieve-by point.

\section{B. TRACON Operations}

Following preconditioning, initial descent, and initiation of FIM operations for equipped aircraft, aircraft should nominally arrive at the TRACON boundary within their speed control margin for correcting any remaining schedule errors and adjusting for disturbances due to winds or other factors inside the TRACON. IM-TAPSS provides TRACON controllers with CMS tools that convey TMA-TM schedule information, progress of aircraft toward their STAs, and speeds that controllers can issue to put aircraft on schedule. Figure 2 illustrates the appearance of the CMS tools in the IM-TAPSS prototype implementation.

The CMS tools include, first, a schedule timeline that shows the TMATM ETAs on the left side, and STAs on the right, for all aircraft at the scheduling point. Filled-in weight-class indicators next to each aircraft identifier mean the associated aircraft's STA is frozen. Second, slot markers (or 'slot-marker circles') translate the schedule information into a spatial target using trajectory predictions through the forecast wind field along each aircraft's assigned OPD. The slot markers show where each aircraft should be now if it were to fly the nominal speed profile and arrive on schedule. The slot markers also show the current indicated airspeed of

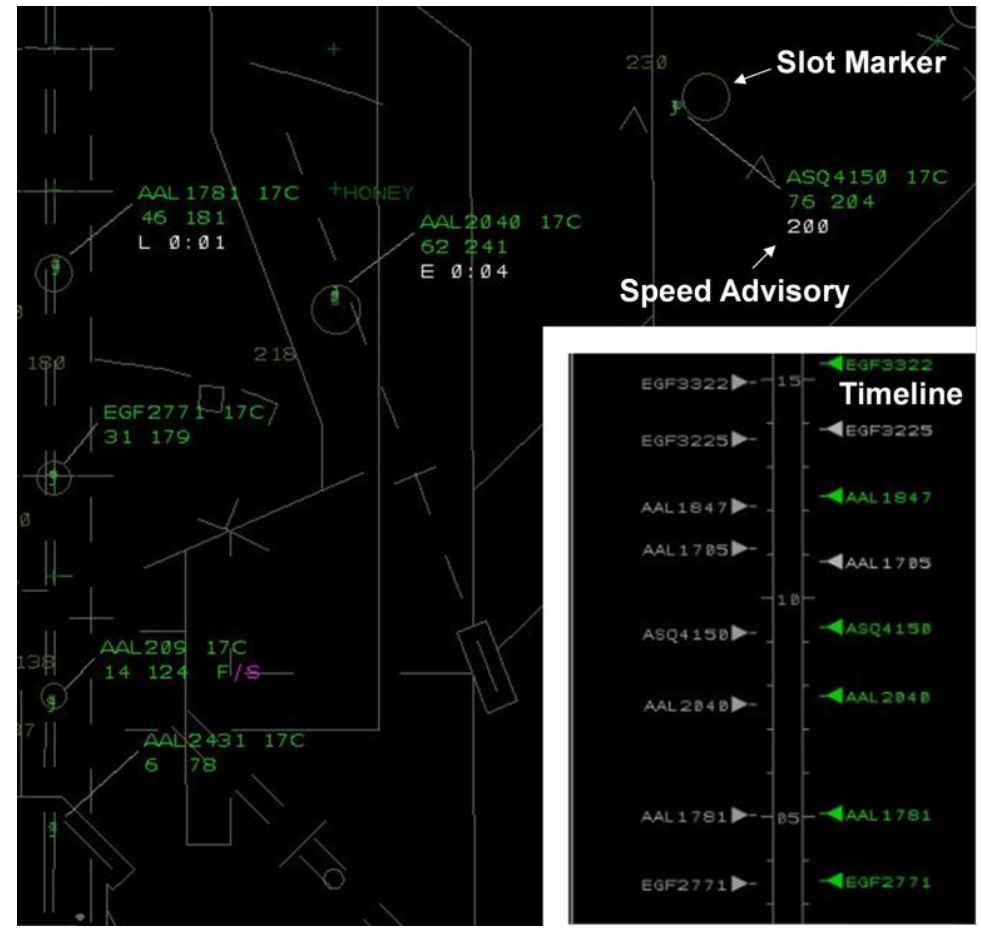

Figure 2. CMS tools. 
the slot marker. Dwelling on aircraft's datablock highlights its slot marker and timeline entries. Finally, the CMS tools include a speed advisory in the third line of the aircraft's data block; the advised speed is predicted to put the aircraft back on schedule. If an advised speed cannot be computed, either because the required speed is outside the available speed control margin or the aircraft is already estimated to be on-schedule, an early/late indicator appears instead of an advised speed. The precise form of the speed advisories remains a topic of ATD-1 research.

Under ATD-1 controllers are expected to use the CMS tools to issue speeds that will yield proper inter-arrival spacing while enabling aircraft to continue flying their assigned OPDs (e.g., <CALLSIGN>, DESCEND VIA THE MAIER THREE ARRIVAL, EXCEPT MAINTAIN TWO-ZERO-ZERO KNOTS). Aircraft conducting FIM operations are expected to notify TRACON controllers upon initial contact (e.g., <CALLSIGN> PASSING ONETWO THOUSAND, INTERVAL SPACING) and continue to follow their FIM speed commands.

\section{Amendments, Interventions, and FIM-Status Reporting}

The ATD-1 ConOps specifies phraseology for handling situations outside the nominal operations outlined above. First, because controllers retain separation responsibility, a controller may find it necessary to terminate an aircraft's OPD. In this situation, standard vectoring phraseology should be used: $\angle$ CALLSIGN>, DESCENT CLEARANCE CANCELLED, FLY HEADING TWO-ZERO-ZERO, MAINTAIN SEVEN THOUSAND FEET.

The ConOps also makes provisions for amending, suspending, resuming, and canceling FIM operations. Individual FIM clearance parameters may be changed with phraseology such as: $\langle$ CALLSIGN>, AMEND INTERVAL SPACING CLEARANCE, SPACE ONE-THREE-FIVE SECONDS BEHIND TARGET. A controller may also temporarily suspend FIM operations and issue a speed clearance to override the ASTAR speed command: <CALLSIGN>, SUSPEND INTERVAL SPACING, SLOW TO TWO-THREE-ZERO KNOTS. The controller may subsequently resume FIM operations, leaving all previous FIM parameters in force: <CALLSIGN>, RESUME INTERVAL SPACING. Suspending and resuming FIM operations is preferable to terminating FIM prior to the achieve-by point; however, a controller may opt to terminate the operation with a clearance such as: <CALLSIGN>, CANCEL INTERVAL SPACING, RESUME PUBLISHED SPEED.

The ConOps also requires flight crews to notify air traffic control when they begin or end FIM operations. Upon initiating paired-spacing operations, flight crews should, for example, report: <CALLSIGN>, INTERVAL SPACING BEHIND DELTA EIGHT-SEVEN-SEVEN. If for some reason the ASTAR algorithm becomes unable to provide speed commands, the crew should also contact the controller (e.g., <CALLSIGN>, UNABLE INTERVAL SPACING, NO TARGET AIRCRAFT DATA). The controller should then respond with a descent clearance similar to ones issued to unequipped aircraft: <CALLSIGN>, CANCEL INTERVAL SPACING, DESCEND VIA THE BONHAM FIVE ARRIVAL, EXCEPT MAINTAIN TWO-FOUR-ZERO KNOTS. In situations in which a controller desires information about the status of a FIM operation, or the value of a particular FIM clearance parameter, the controller can query the aircraft (e.g., <CALLSIGN>, REPORT INTERVAL SPACING TARGET).

\section{Summary}

The ATD-1 ConOps synthesizes schedule-based operations supported by CMS tools and ADS-B FIM operations into a NextGen solution for realizing high-throughput, efficient descents at busy airports. As the proportion of FIMequipped aircraft increases, their contributions to improved overall arrival precision are expected to further increase the proportion of uninterrupted OPDs. The tools, procedures, and clearance phraseology specified in the ATD-1 ConOps are subject to refinements as IM-TAPSS matures from a laboratory protoype into a demonstration system. The next section describes the first steps in this process: human-in-the-loop simulation studies in the AOL using initial IM-TAPSS laboratory prototypes.

\section{Simulation Studies}

The AOL uses a suite of MACS workstations to provide faithful emulations of operational Center and TRACON controller workstations, and as psuedo-pilot stations for controlling simulated aircraft. ${ }^{9}$ To support the IM-TAPSS integration effort, eight operator positions for ASTOR single-piloted simulators were installed in a portion of the AOL (Fig. 3). Adding the TMA-TM, along with TMA-TM timeline displays in the Center and TRACON control rooms, readied the AOL for the initial series of CMS

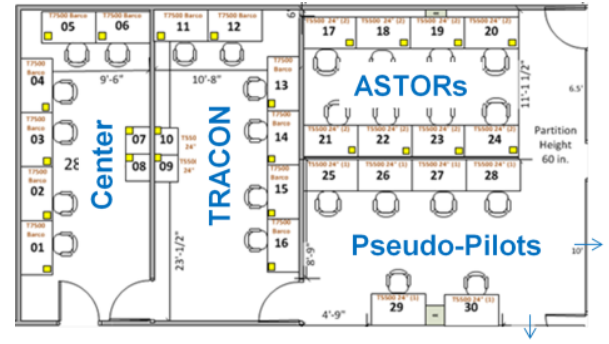

Figure 3. AOL simulation layout. 
ATD-1 simulations. All the simulations used Dallas-Ft. Worth (DFW) airspace, with ASTORs and other MACSsimulated arrival traffic on charted OPDs initially developed at NASA Langley as part of prior FIM research. Traffic scenarios were constructed based on DFW traffic mixes, and designed to support one-hour simulation trials. In all the simulations one retired controller served as a Center 'ghost' confederate responsible for transferring control of aircraft to the Center controllers, and another as the Tower confederate. All assumed IFR operations with standard $3 / 4 / 5 \mathrm{nmi}$ wake-vortex spacing requirements. In all simulations TMA-TM added a $0.3 \mathrm{nmi}$ scheduling buffer to the standard wake-vortex spacing minimums. TRACON controllers could use Terminal Proximity Alert (TPA) spacing cones to assess relative spacing between aircraft. This section first briefly describes the CA-1 and CA-2 studies and their findings. It then describes the most recent CA-3 simulation and presents its results in detail.

\section{A. CA-1 and CA-2 Simulations}

CA-1 followed the initial integration step in which IM-TAPSS components were integrated in the AOL. Simulated OPD arrivals crossed three meter fixes on the TRACON boundary and merged to land on DFW runway $17 \mathrm{C}$ (Fig. 4). A mix of general aviation students and pilots flew the eight FIM-equipped ASTORs, and retired air traffic controllers staffed three en-route and three TRACON sectors; the TRACON controllers had all participated in previous CMS simulations. After an initial training day, eighteen data-collection trials were conducted in both baseline and 'ATD' conditions. CA-1 demonstrated the critical importance of preconditioning all arrivals. The results of the study also showed controllers were receptive to the mixed-FIM-equipage operations, and found workload and the proposed clearance phraseology acceptable. They also gave the CMS tools usability ratings in line with prior CMS research, with slot-marker usability rated highest. ${ }^{10}$

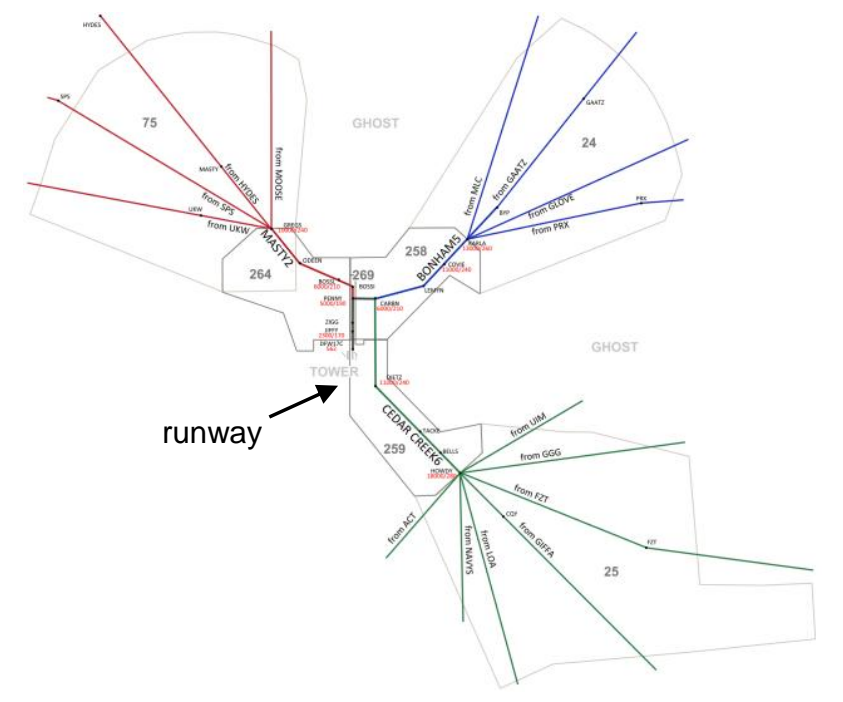

Figure 4. CA-1 and CA-2 airspace and routes to DFW runway 17C.

CA-2 reused the CA-1 study airspace to focus on en-route pre-conditioning of both FIM- and non-FIM-equipped aircraft. Traffic scenarios were refined and the routes used by MACS, TMA-TM and the ASTOR simulators were harmonized. The CA-2 controllers had all participated in CA-1, as had many ASTOR pilots; on the whole, the eight CA-2 ASTOR pilots were better qualified. Again one day of training was followed by eighteen data-collection trials designed to compare baseline (non-FIM, current-day control) operations to operations under various tool configurations, both in the Center and TRACON. The standard CMS tools comprised the TRACON toolset. In the Center, controllers used meter lists with times to their respective meter fixes displayed with either one-minute or one-second precision. One condition included en-route speed advisories, while another added en-route slot markers.

An important addition to both CA-1 and CA-2 was a FIM-status designator controllers could add to a FIMequipped aircraft's data block. If after receiving the FIM clearance the ASTOR pilot reported ASTAR was commanding speeds to the assigned RTA, controllers could toggle an ' $\mathbb{R}$ ' at the end of its data block's second line; once the pilot reported ASTAR was engaged in paired-spacing mode, controllers could remove the $₫$ and replace it with an ' $\mathrm{S}$ ' to remind themselves and advise downstream controllers that the aircraft was currently conducting FIM operations. CA-2 included experimental conditions in which slot markers and speed advisories dropped off when controllers entered a FIM-status designator.

En-route pre-conditioning of all aircraft in CA-2 alleviated problems observed in CA-1 by helping keep required TRACON adjustments within achievable speed-control margins for both FIM and non-FIM aircraft. Meter-fix delivery accuracy improved when en route controllers had delays indicated to one-second precision. While en-route slot markers did not improve delivery accuracy, controllers found them useful in cases where path changes were needed for pre-conditioning. CA-2 again found CMS tools advantageous for maintaining OPDs and reducing the variation of inter-arrival spacing at the runway threshold. CMS tool ratings mirrored those of prior CMS studies, ${ }^{5}$ with TRACON slot markers rated best. Both Center and TRACON controllers found the FIM-status designators useful. Again in CA-2 workload was always manageable, but the CMS tools yielded a small but statistically significant reduction in TRACON workload ratings. 


\section{B. CA-3 Simulation}

CA-1 and CA-2 were invaluable for addressing IM-TAPPS systems-integration issues and exploring ATD-1 operations with humans in the loop. CA-3 built on these efforts to evaluate IM-TAPPS 'Version 1' controller tools, ConOps procedures, and ConOps phraseology. For CA-3, the DFW study airspace was expanded, OPDs updated with new versions from NASA Langley, and procedures adapted to ensure aircraft were preconditioned as early as possible. Center controllers were provided with speed-advisory functionality in line with proposed FAA en-route modernization plans. The FIM-status designators and CMS speed advisories were also modified, and FIM operations were specified to use the FAF as the achieve-by point. CA-3 also included actual and forecast winds for the ASTORs, TMA-TM and MACS. Perhaps the most noteworthy change from CA-1 and CA-2, however, was that the CA-3 controller participants had no prior experience with the CMS tools or the MACS simulation platform. The following subsections describe the CA-3 study from a ground-side perspective.

\section{Routes, Airspace, and Traffic Scenarios}

Simulated aircraft arrived on RNAV OPDs across four meter fixes (Fig. 5). All included approach transitions that connected to instrument approach procedures. East-side OPDs merged to DFW runway 17C, while west-side OPDs merged to runway $18 \mathrm{R}$; operations to runways $18 \mathrm{R}$ and $17 \mathrm{C}$ were independent. The OPDs were developed at NASA Langley for ATD-1 FIM research by adapting existing DFW arrival procedures. The altitude and speed restrictions produce consistent vertical and speed profiles along the different lateral routes. The merge-point and initial-approach-fix altitude restrictions were designed to provide altitude separation, and speed restrictions at those points were designed to limit overshoots on final-approach intercept.

The study airspace included four en-route arrival sectors, two east-side Feeder sectors, two west-side Feeder sectors, and two Final sectors. AOL researchers constructed traffic scenarios by distributing recorded DFW arrivaltraffic mixes to the routes so that the different sectors experienced comparable traffic loads. Two baseline scenarios

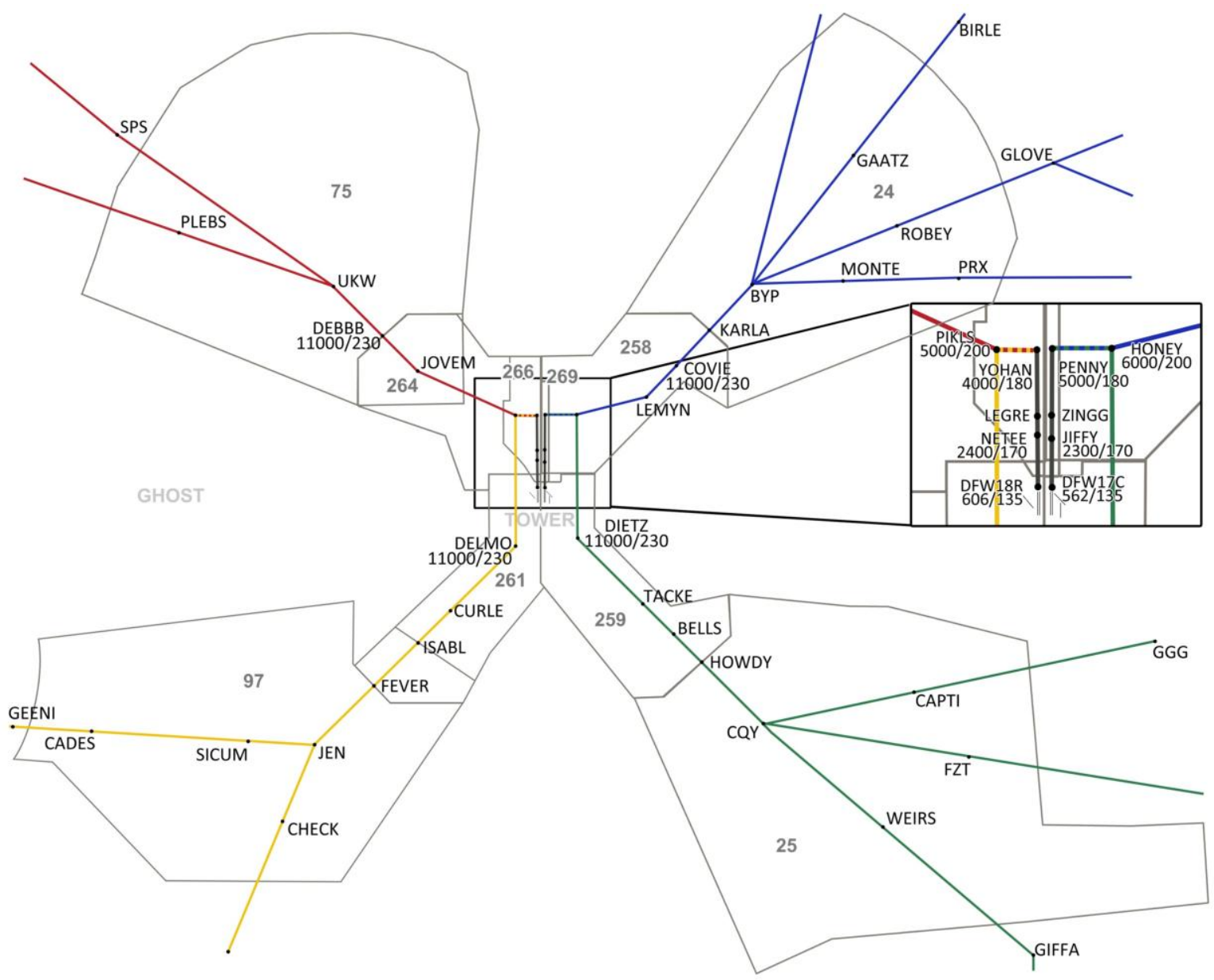

Figure 5. CA-3 airspace and routes to DFW runways $17 \mathrm{C}$ and 18R.

American Institute of Aeronautics and Astronautics 
were first developed. Two variations (' $a$ ' and ' $b$ ') of each scenario were then created by selecting eight aircraft to replace with the eight ASTOR simulators. In the resulting scenarios, two ASTORs crossed each meter fix sequenced for arrival in 'strings' of various lengths. This gave Center controller participants the opportunity to precondition and issue FIM clearances to the same number of ASTORs over the course of CA-3, and gave ASTORs the opportunity to space behind other ASTORs or MACS aircraft flying the same or different OPDs.

\section{Winds}

For CA-3, researchers added forecast winds to the TMA-TM and both actual and forecast winds to MACS and the ASTOR simulators. Figure 6 shows a simple linear forecast-wind profile, together with a piece-wise linear actual-wind profile, used to specify two wind conditions: 'Winds 1' out of $165 \mathrm{deg}$, and 'Winds 2' out of $195 \mathrm{deg}$. The winds and wind-forecast errors are relatively benign, but sufficient to demonstrate consistent operation of the IM-TAPSS laboratoryprototype components in the presence of winds and observe any unusual effects of winds on preconditioning, FIM operations, and TRACON merging and spacing using the CMS tools.

\section{Participants}

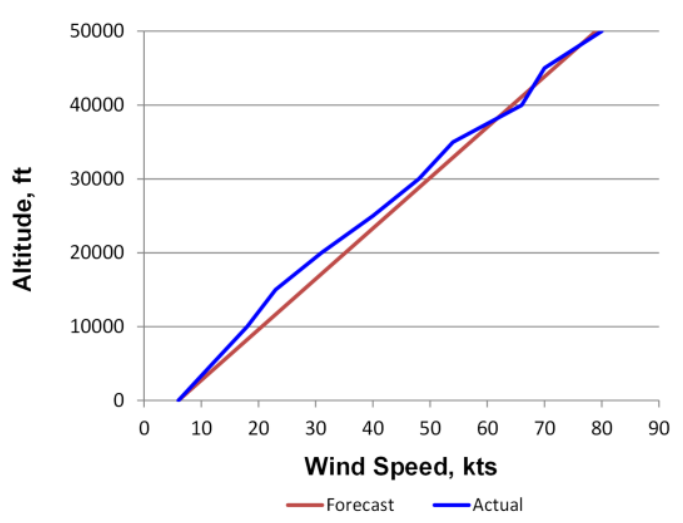

Figure 6. CA-3 forecast and actual wind profiles.

DFW controllers who had been retired an average of 1.8 years (1 month-5.3 years) participated in CA-3. All were highly experienced, with nearly 30 years of experience on average. Controllers staffed positions according to their specializations: the four en-route controllers had specialized in the Center domain for their entire careers, the Feeders had an average of 17 years of specialization, and the Finals had an average of 24 years of specialization. Controllers remained on the same position during the data-collection portion of the study. The controllers knew the airspace well and showed some degree of familiarity with the operational concept, but none had any previous experience with the CMS tools, FIM operations, or the MACS simulation platform used in the AOL.

ASTOR pilots were all Boeing glass-cockpit type-rated pilots, most of whom had participated in CA-2. Personnel from NASA Langley were on-hand to provide training support. As in the previous simulations, general aviation pilots and students with AOL pseudo-piloting experience staffed the MACS pseudo-pilot stations.

\section{Training}

AOL researchers conducted training for the last three days of the week prior to data-collection. Training began with a general briefing about the study, followed by separate briefings for pilots and controllers. The next two-andone-half days were devoted to simulation runs intended to familiarize the controllers with MACS and the CA-3 operations. Controllers started with light-traffic training runs while the ASTOR pilots practiced separately. The training progressed to include ASTORs conducting FIM operations and increase traffic levels. Training concluded with a review briefing.

\section{Controller Tools}

In conditions with controller tools, the TRACON controller workstations were configured with the CMS tools shown in Fig. 2. In CA-3 the speed advisories showed a speed to fly on the current speed segment of the OPD. When an aircraft was within approximately $3 \mathrm{nmi}$ of its OPD's next speed segment, the advised speed changed to one computed based on the next speed segment. Controls on the CMS timeline displays that support schedule adjustments (e.g., STA swaps) were enabled. MACS controller workstations also included other currently-deployed functionality (e.g., J-rings).

Center controller workstations included meter lists to support preconditioning. In baseline 'Current-Day' trials, the meter lists appeared as shown in Fig. 7; the reference fix is listed at the top, and delay-countdown values are displayed with one-minute precision. Dwelling on a meter-list entry or an aircraft's data block highlights both. The meter lists in 'Tools' conditions (Fig. 8) work similarly, except they show delay countdown with one-second precision, and when a FIM-equipped aircraft's STA freezes, FIM-clearance information derived from TMA-TM schedule is also displayed. For the dwelled aircraft in Fig. 8 (AAL846), the meter list gives the FAF 'NETEE' as the achieve-by point with an RTA at 1749.18Z, and an advised spacing interval of $117 \mathrm{~s}$ behind target AAL9830 flying the BOWIE ONE arrival, TEXICO transition. 


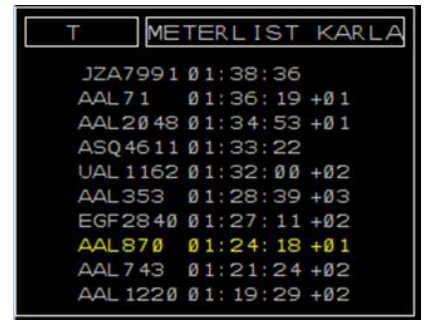

Figure 7. Current-day meter list.

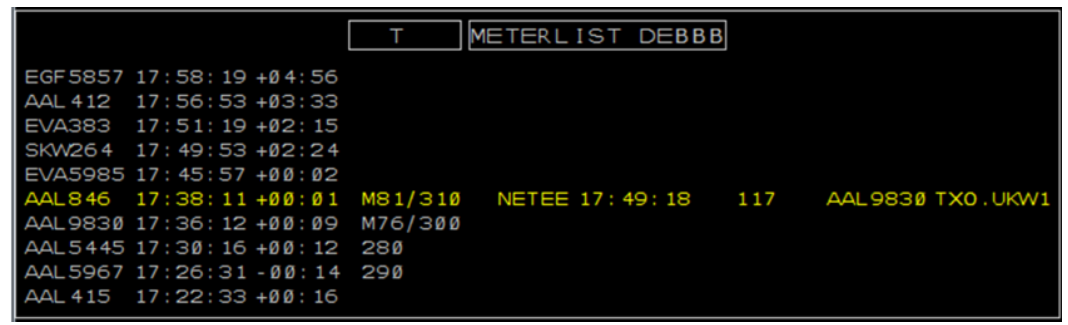

Figure 8. Tools-condition meter list with advised speeds, FIM-clearance information, and delay values with one-second precision.

Figure 8 also shows how advised/issued speeds appear in the meter list in Tools conditions. As shown in Fig. 9, an ' $\mathrm{I}$ ' indicates in an en-route speed advisory is available; a data block portal enables en-route controllers to access fly-out menus for selecting speeds to issue to meet STAs at the meter fix. Figure 9 also illustrates an early/late indication, which was displayed below each aircraft's target symbol in CA-3.

FIM-status designators for CA-3 were also modified slightly from the prior simulations. Aircraft equipped for FIM operations were identified with a '/S.' When the controller issued the FIM clearance, an 'IM' command was used to turn the ' $/ \mathrm{S}$ ' magenta, and add the ${ }^{\circledR}$ after it. These symbols could then be independently toggled to display current

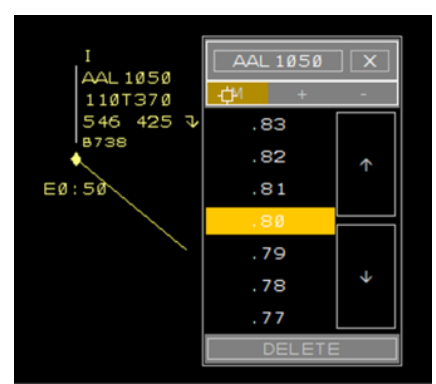

Figure 9. Fly-out menu for enroute speed-clearance entries.

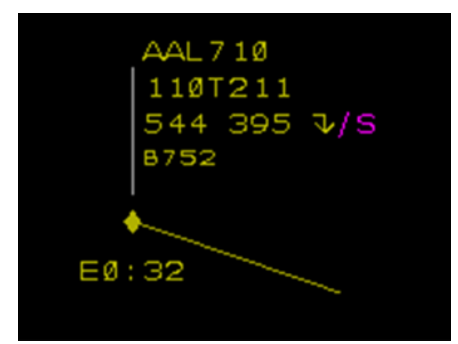

Figure 10. Data block with paired-spacing FIM status.

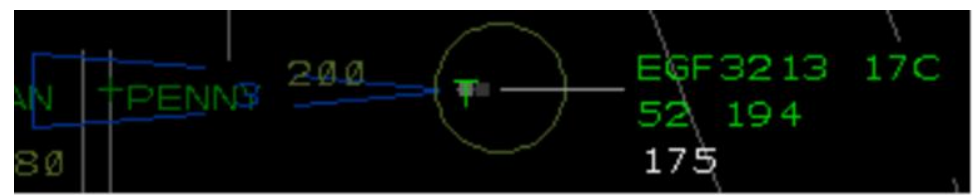

Figure 11. Spacing cone displayed for an aircraft together with a CMS slot marker and speed advisory. FIM status. Figure 10 shows a FIM-

equipped aircraft designated to be actively conducting FIM operations in paired-spacing mode. The FIM-status designators transferred to downstream-controller workstations, so TRACON controllers could cross-check pilot FIM-status reports with their last-reported en-route FIM status. TRACON controllers were free to display a TPAtype spacing cone for any aircraft by entering a command that included the desired cone length. As emulated in MACS, the cones appear as shown in Fig. 11.

\section{Procedures and Phraseology}

Controller training focused on ATD-1 procedures and clearance phraseology in keeping with the ConOps. As aircraft arrived in cruise, the Center ghost controller shipped aircraft to the Center controllers as early as practicable, so that they could begin preconditioning aircraft as soon as their STAs froze. Center controllers were asked to prefer delivering aircraft to the TRACON a few seconds early with a current speed near their OPD speed profile, to avoid situations in which aircraft later require speed increases in the TRACON. Once the aircraft were preconditioned, the Center controllers issued FIM clearances to the FIM-equipped ASTORs. An outstanding integration issue additionally required the controllers to ensure aircraft were reestablished on their original RNAV routes (i.e., rather than a routing direct to the meter fix) before issuing the FIM clearance; otherwise, the ASTAR algorithm would not engage until the aircraft returned to the original RNAV route.

Briefings emphasized phraseology, and controllers were provided with a list of expected pilot and controller communications. The nominal controller phraseology conformed to the examples presented above in Section II. To prepare pilots for the FIM clearance, controllers prefaced it with: <CALLSIGN>, INTERVAL SPACING AVAILABLE, ADVISE WHEN READY TO COPY. The FIM achieve-by point was specified to be the FAF (i.e., NETEE or JIFFY), and was established as the same as the RTA reference point, so that the FIM clearance was phrased as (using the meter-list information in Fig. 8 as an example): AMERICAN 846, FOR INTERVAL SPACING, CROSS NETEE AT 1719 PLUS 18. WHEN ABLE, SPACE 117 SECONDS BEHIND AMERICAN NINE-EIGHT-THREE-ZERO ON THE BOWIE ONE ARRIVAL, TEXICO TRANSITION. 
Pilots were to read this clearance back as: SCHEDULED TIME AT NETEE IS 1719 PLUS 18 . SPACE 117 SECONDS BEHIND AMERICAN NINE-EIGHT-THREE-ZERO ON THE BOWIE ONE, TEXICO TRANSITION, AMERICAN 846. If they deemed it necessary to intervene, Center and TRACON controllers were encouraged to suspend the FIM clearance rather than cancel it, if possible, so that pilots might later be able to resume FIM operations to the FAF. Pilots were expected to report when they were following FIM-commanded speeds in paired-spacing mode, and whether their FIM status changed. Controllers could then update the aircraft's FIM-status designator accordingly.

TRACON controllers were briefed to keep aircraft on their RNAV OPDs to the extent possible, and coordinate with each other as necessary. To facilitate coordination, controllers in the TRACON room were situated with the Final controllers seated next to each other, the low-side (northern) Feeder controllers seated next to their respective Finals, and the high-side (southern) Feeder controllers next to them. When the CMS tools were available, the controllers were asked to attempt to use and evaluate them. Training also emphasized that the CMS speed advisories present suggested speeds that controllers should feel free to modify to suit the situation. The Final controllers were asked to issue speeds as necessary to achieve proper spacing at the FAF, considering compression thereafter, before transferring control to the Tower confederate.

\section{Experimental Design and Schedule}

CA-3 was designed to compare 'Baseline' operations (during which the ASTORs participated, but did not conduct FIM operations) with a 'Tools' condition in which the TRACON controllers had CMS tools available, the Center controllers had enhanced meter lists and speed advisories, and the ASTORs conducted FIM operations. The data-collection schedule is shown in Fig. 12 (asterisks indicate aircraft callsigns were altered from the previous scenario). The experimental design replicates the Tools conditions, so participants experienced each scenario and wind condition once under Baseline conditions and twice with tools. Each experimental trial lasted approximately one hour, after which participants filled out online questionnaires tailored to the preceding trial. Participants answered a comprehensive questionnaire prior to the closing debriefing.

\begin{tabular}{|c|c|c|c|c|}
\hline DAY 1 & DAY 2 & DAY 3 & DAY 4 & DAY 5 \\
\hline \multicolumn{5}{|c|}{ Briefing / Comm Checks } \\
\hline Run 1 & Run 6 & Run 11 & Run 16 & Run 21 \\
\hline Baseline & Tools & Tools & Baseline & Tools \\
\hline Scenario 1a & Scenario $2 b$ & Scenario 1a & Scenario $1 \mathrm{~b}$ & Scenario $2 a$ \\
\hline & & & & \\
\hline \multicolumn{5}{|c|}{ Questionnaires/Break } \\
\hline Run 2 & Run 7 & Run 12 & Run 17 & Run 22 \\
\hline Tools & Tools & Baseline & Tools & Tools \\
\hline Scenario 2a & Scenario $1 \mathrm{~b}$ & $\begin{array}{l}\text { Scenario 2b } \\
\text { Winds } 2\end{array}$ & Scenario $1 \mathrm{~b}^{*}$ & $\begin{array}{l}\text { Scenario 1b } \\
\text { Winds } 1\end{array}$ \\
\hline \multicolumn{5}{|c|}{ Questionnaires/Break } \\
\hline Run 3 & Run 8 & Run 13 & Run 18 & Run 23 \\
\hline Tools & Baseline & Tools & Tools & Baseline \\
\hline Scenario 1b & Scenario $1 \mathrm{a}$ & Scenario $2 a$ & Scenario $2 b$ & Scenario $1 b^{*}$ \\
\hline Winds 2 & Winds 1 & Winds 1 & Winds 2 & Winds 2 \\
\hline \multicolumn{5}{|c|}{$\begin{array}{l}\text { Questionnaires } \\
\text { Lunch Break }\end{array}$} \\
\hline \multirow{4}{*}{$\begin{array}{l}\text { Baseline } \\
\text { Scenario } 2 b \\
\text { Winds } 1\end{array}$} & Run 9 & Run 14 & Run 19 & Run 24 \\
\hline & Tools & Baseline & Tools & Tools \\
\hline & Scenario $2 a$ & Scenario $2 a^{*}$ & Scenario 1a & Scenario $2 b$ \\
\hline & Winds 2 & Winds 2 & Winds 2 & Winds 1 \\
\hline & & \multicolumn{3}{|c|}{ Questionnaires/Break } \\
\hline \multirow{3}{*}{$\begin{array}{c}\text { Run } 5 \\
\text { Tools } \\
\text { Scenario 1a } \\
\text { Winds } 1\end{array}$} & Run 10 & Run 15 & Run 20 & Questionnaires \\
\hline & Scenario $2 b$ & Scenario 1a & Scenario 2a & \multirow{3}{*}{ Debrief Discussion } \\
\hline & Winds 1 & Winds 1 & Winds 1 & \\
\hline \multirow{2}{*}{\multicolumn{4}{|c|}{$\begin{array}{c}\text { Questionnaires } \\
\text { Wrap-up Discussion }\end{array}$}} & \\
\hline & & & & \\
\hline
\end{tabular}

Figure 12. CA-3 data-collection schedule.

\section{Data Collection}

In addition to the post-trial and post-simulation questionnaire responses, all MACS and ASTOR stations logged digital data, including flight state information, pilot and controller entries, and schedule information. TMA-TM also logged digital data. AOL researchers stationed in the Center and TRACON control rooms and the ASTOR area of the AOL observed the operations. In addition, screen-capture movies that include recorded audio were collected from all MACS and ASTOR stations. The next subsection presents the results of the CA-3 simulation.

\section{CA-3 Results}

The IM-TAPSS Version 1 laboratory prototype was reliable and robust to the actions of the novice controllers, enabling AOL researchers to examine data from all the system components for all twenty-four experimental trials. To prepare for analyzing future ATD-1 integrated simulations, the analysis sought to extend the ground-side-focused data analyses used in prior CMS research to address ATD-1 operations with both MACS and ASTOR aircraft participating in the simulation. The following subsections first present general arrival metrics, followed by results that address specific aspects of the controller tools, procedures, and phraseology. Except where noted, the analyses focus on the set of 1,124 aircraft that reached the runway threshold. During Tools conditions, 128 ASTORs were eligible to conduct FIM operations. 


\section{Throughput}

Figure 13 shows the average throughput over the simulation trials per runway in the two conditions (error bars represent one standard deviation). Throughput did not differ significantly between the two runways in the Tools and Baseline conditions, and no effects due to scenario or winds were observed. However, across scenarios the TMA-TM schedules for $18 \mathrm{R}$ were observed to include a few small gaps, which could account for the higher throughput on 17C. Runway throughput differences were significant in conditions with tools $(\mathrm{p}<0.02)$.

Average throughput was also computed using ten-minute time windows at each minute after the first aircraft arrived at the runway threshold (Fig. 14). This analysis suggests that, on average, controller tools provided a slight advantage for sustaining throughput levels during the middle 15-30 minutes of the arrival rush.

\section{Route Conformance}

The only means of precisely determining how often controllers interrupted OPDs is to listen to the clearances issued by each controller. Route-conformance analyses examined RNAV path conformance, level-offs, and speed-restriction compliance as surrogate measures. The TRACON controllers were for the most part successful in keeping aircraft on their lateral routes in both conditions; 12 path clearances were issued in Baseline conditions, while 16 path clearances were issued in Tools conditions (some of these involved aircraft that had failed to properly comply with prior clearances). However, most of the controllers commented that they would use vectors more in the real world-with speeds to maintain spacing once it was established - and that they concentrated on using speed control more than they normally would have. One controller liked not having to worry about the lateral path, while another commented that issuing too many speeds "conveys a message that you don't know what you're doing." Both Final controllers commented that they had to monitor speeds more closely than they normally would.

An analysis of level-flight segments in the TRACON below 11,000 ft found the profiles of 24 unique aircraft had level segments at least 2 nmi long under Baseline conditions; thirteen of these were ASTORs. Twenty aircraft were found under Tools conditions; five were ASTORS. Almost all these level segments were detected between 5,000 and 7,000 ft, when aircraft were in or near the base-turn region.

Aircraft were also occasionally detected with speed errors in excess of $20 \%$ at speedrestriction points (Fig. 15). These appear to be principally due to flight-deck energy management issues, rather than controllers issuing considerably different speeds from those on the charted OPDs. Only three ASTOR simulators had excess speed errors in the Tools

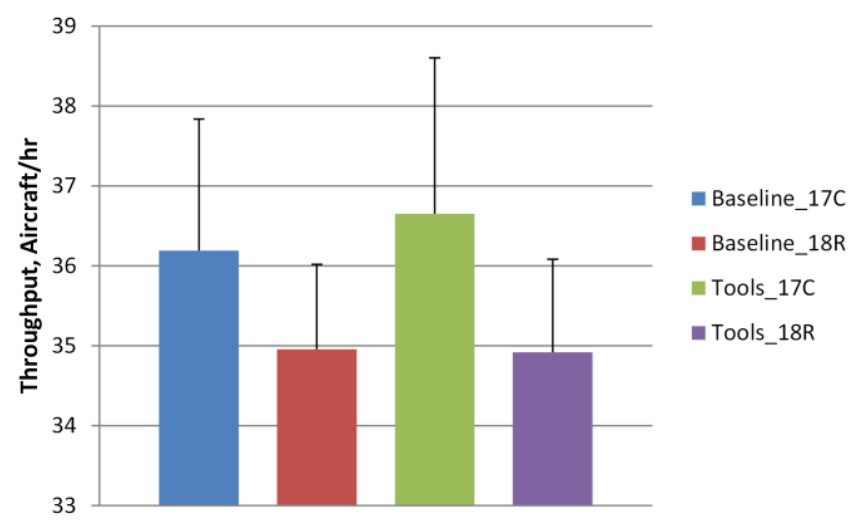

Figure 13. Average throughput by runway and condition.

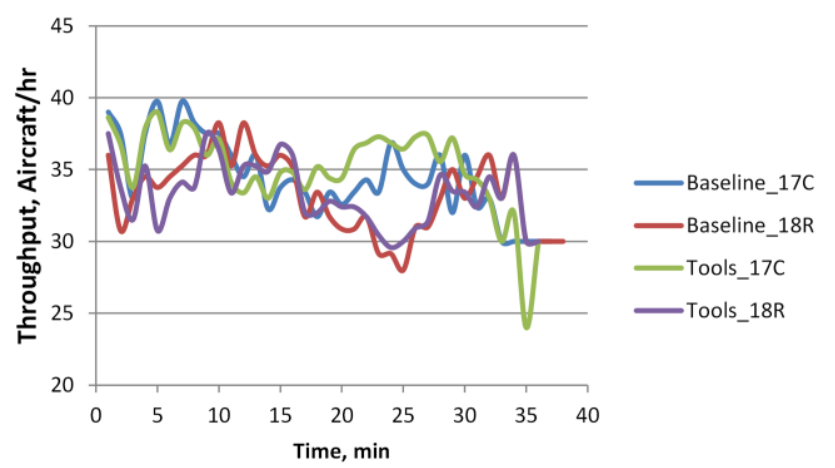

Figure 14. Average throughput during ten-minute windows.

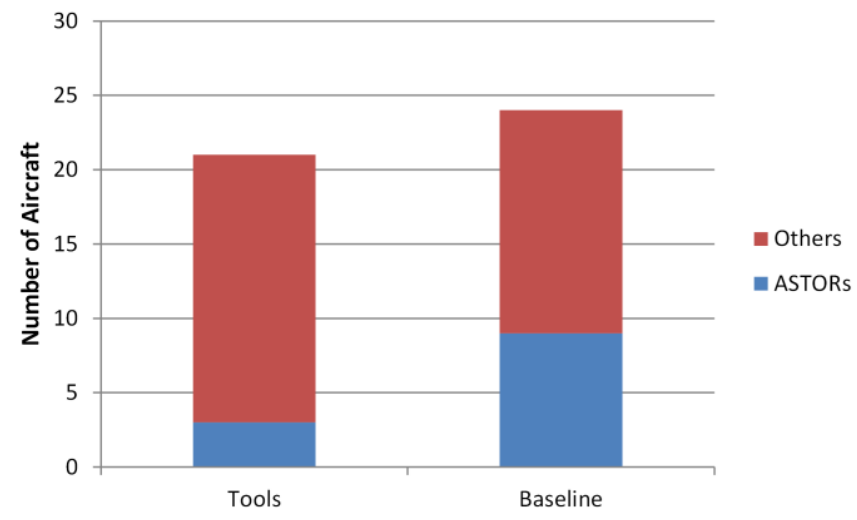

Figure 15. Speed errors greater than $20 \%$ at speed restrictions in all trials by simulator type and condition.

10

American Institute of Aeronautics and Astronautics 
conditions, when ASTORs were largely following FIM speed commands.

Route conformance was also analyzed as it pertained to preconditioning aircraft in the Center. Center controllers exhibited considerable individual differences in their use of path clearances (Fig. 16). However, all used more path clearances in the Tools conditions. One possible explanation for this is that the higher-precision meter-list delay values in the Tools conditions compelled the controllers to work harder to precondition aircraft; however, post-trial questionnaire responses about en-route vectoring offer a variety of reasons. These ranged from vectoring for separation because speed-control would not resolve conflicts quickly enough, 'problem' aircraft affecting

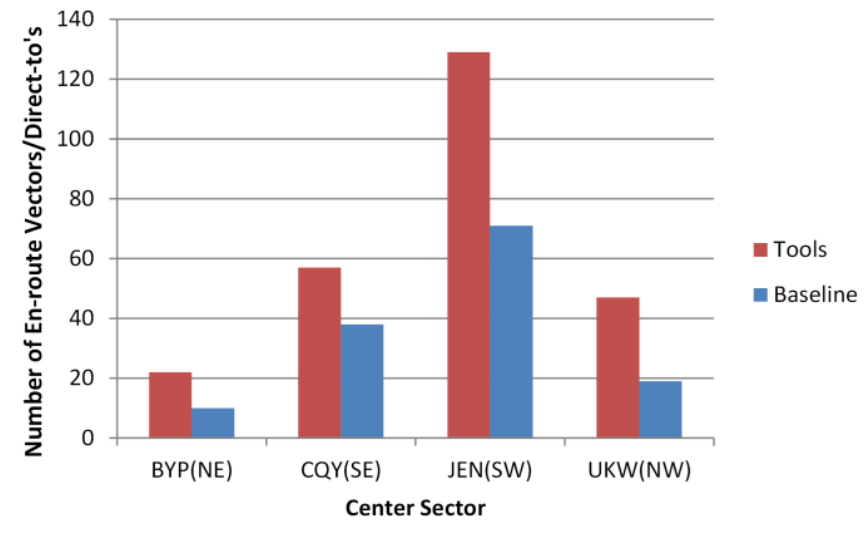

Figure 16. Number of path clearances issued by each Center controller in all trials by condition. subsequent aircraft, using 'direct-to' clearances to make up time, and simply deciding to try using path instead of speed.

\section{Flight Time and Distance}

An analysis of overall aircraft flight time and distance showed no significant differences between flight times or distances between the Tools and Baseline conditions. Wind conditions led to significant differences in both flight time and distance only for Baseline trials ( $\mathrm{p}$ 0.04). Within the TRACON, flight time and distance did not differ significantly. However, when measured starting from inside the two merge points (i.e., PIKLS and HONEY), a slight but significant increase in flight time was measured for the Baseline trials $(\mathrm{p}<0.001)$. This result is in line with observations that, in Baseline trials, controllers seemed to issue slower speeds to fly in the base-leg region to avoid vectoring aircraft to the final approach. The flight-time difference equates to an average ground speed of 159 kts from the merge points to the runway thresholds in the Tools conditions versus $156 \mathrm{kts}$ in the Baseline conditions. Within the Tools conditions, average flight time and distances starting from inside the merge points were different in the two wind conditions $(\mathrm{p}<0.03)$.

\section{Arrival Spacing Accuracy}

The distance to each arrival's trailing aircraft was measured as it crossed its FAF, and the required wake-vortex spacing distance was subtracted for each pair. The resulting inter-arrival spacing errors are shown in the histogram in Fig. 17. One violation was detected at the FAF; it occurred in a trial with tools when an ASTOR following speed commands in RTA mode became too close to an unequipped aircraft arriving slightly behind schedule. The spacing accuracy achieved in the Tools conditions better compensates for additional compression inside the FAF than that in the Baseline conditions, leading to a $37 \%$ decrease in wake-vortex spacing violations measured at the runway thresholds. Controllers also felt the tools helped them achieve better spacing. The contribution to spacing accuracy of FIM-equipped aircraft operating in paired-spacing mode is shown in Fig. 18. While the paired-spacing aircraft were in many cases following aircraft that were not conducting FIM operations, the FIM operations contribute to a reduction in inter-arrival spacing variation.

\section{Schedule Conformance}

The TMA-TM computed schedules during all simulation trials. While not technically intended for use in the Baseline condition, schedule timelines were also projected on the walls of the control rooms during all trials; this was done so that experimenters could inspect the schedules - but it also enabled controllers to use them (and some did). Figure 19 shows the average required delays on the meter-fix TMA-TM schedules when STAs were first frozen and when aircraft crossed the meter fix, as well as the required delays on the TMA-TM runway schedules when the aircraft crossed the FAF in each condition (error bars represent one standard deviation). None of the differences are significant, but aircraft Center controllers preconditioned aircraft slightly better during trials with tools. Also, the runway-schedule conformance measured at the FAF varied more. As expected, aircraft conducting FIM operations conformed to the schedule to the extent their targets did; the two ASTORs that arrived at the FAF in RTA mode conformed closely to the schedule. 


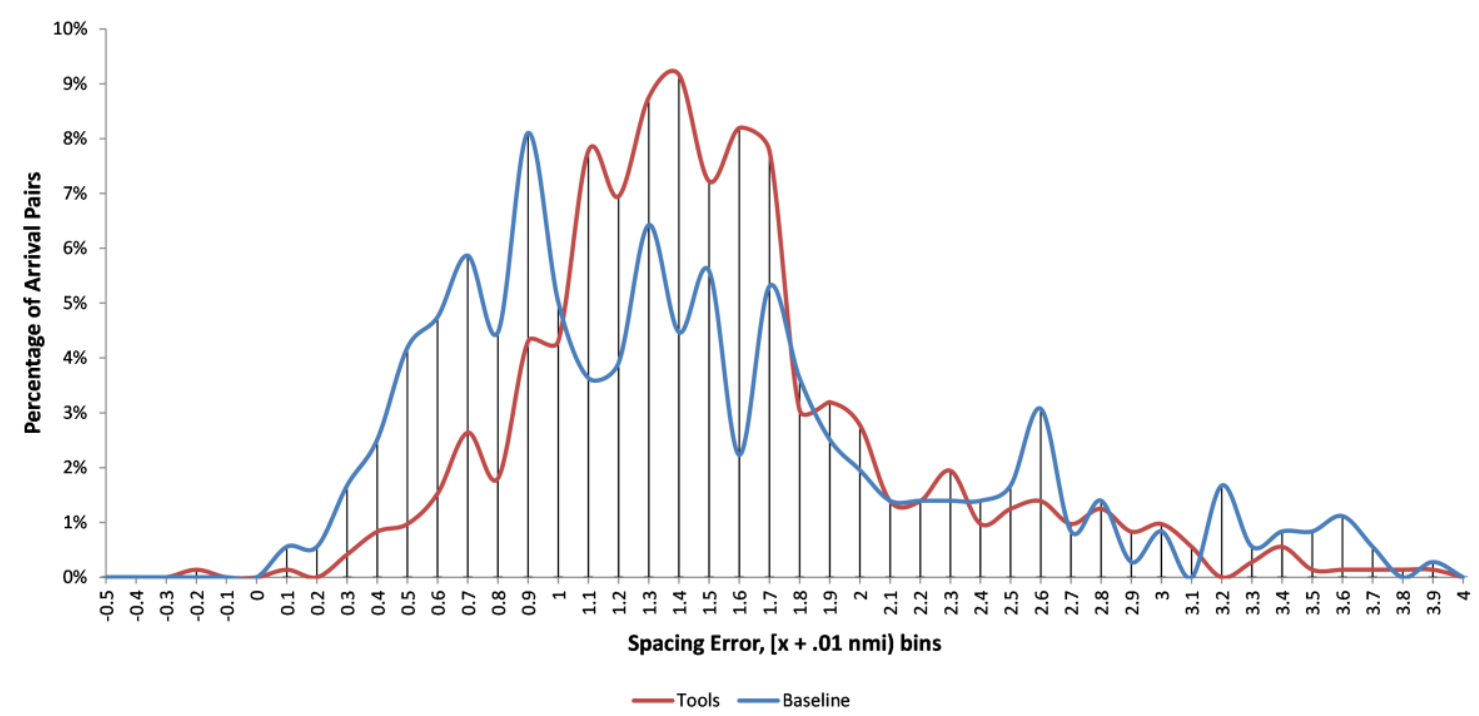

Figure 17. FAF spacing relative to required wake-vortex spacing by condition.

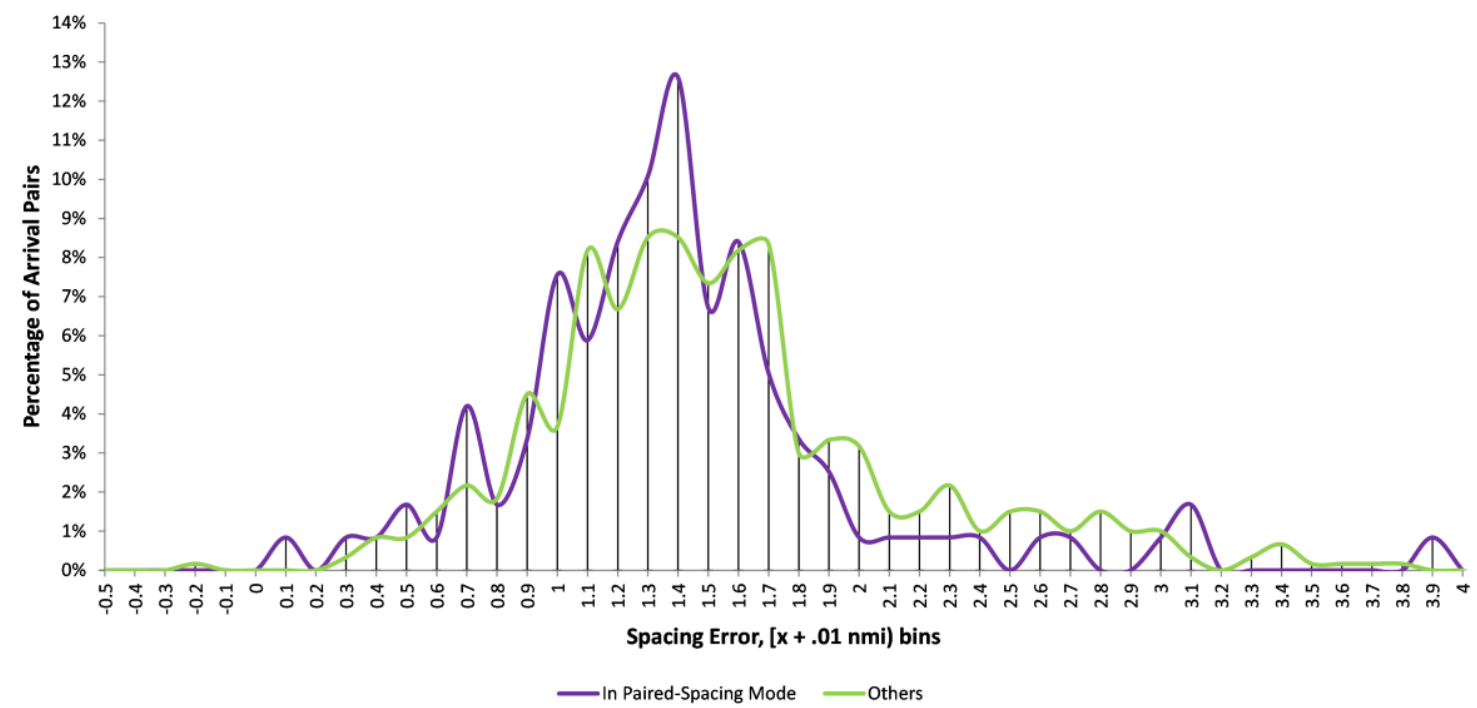

Figure 18. FAF spacing relative to required wake-vortex spacing for FIM-equipped aircraft in paired-spacing mode versus other aircraft in Tools conditions.

\section{FIM Operations}

Of the 128 ASTORs eligible to conduct FIM operations in the Tools conditions, all received FIM clearances. Of these, 116 successfully arrived at the FAF in pairedspacing mode - a $91 \%$ overall success rate for FIM-equipped aircraft. Two arrived in RTA mode and five were no longer performing FIM. ASTOR fuel burn did not differ significantly between the Tools and Baseline conditions, nor did it differ for the different wind conditions in conditions with tools.

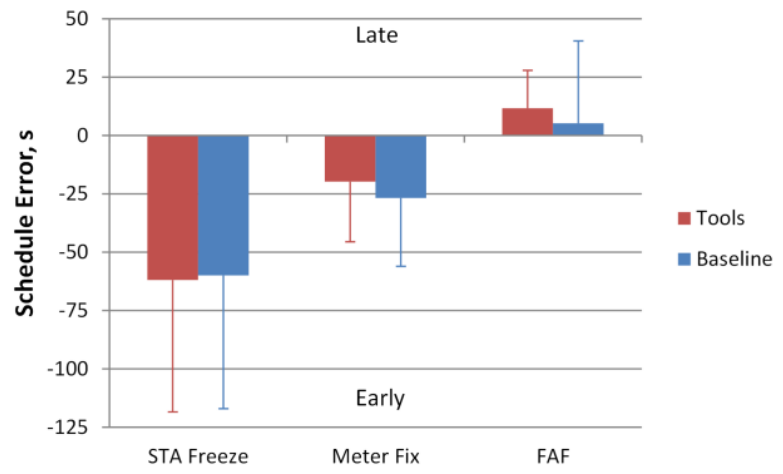

Figure 19. Schedule conformance by location and condition.

12

American Institute of Aeronautics and Astronautics 
Controllers overwhelmingly agreed that mixed-FIM-equipage operations were 'not a problem.' One commented this would be especially true once everyone got used to the procedures and phraseology. Two noted they saw no alternative to mixed-FIM-equipage flows - at some point the aircraft would have to merge. In approximately twothirds of the trials with tools, controllers noted no problems. However, of reported problems, aircraft conducting FIM operations comprised the largest category (23\%); FIM-equipped aircraft in RTA mode were cited in $7 \%$ of reports. More than half the time controllers did not have to change the way they worked to accommodate FIM operations; however, $20 \%$ of the time they reported changing their general scan, $16 \%$ of the time they reported issuing more clearances, and $12 \%$ of the time they reported issuing different clearances than usual.

AOL researchers conducted an exhaustive analysis of the FIM clearances controllers issued by listening to the pilot-controller voice communications. On average, FIM operations commenced $2 \mathrm{~min} 47 \mathrm{~s}$ after the controllers advised the pilots that a FIM clearance was available (48 s-6 min 8 s). Digital-data processing enables visual detection of FIM interruptions due to vectoring; analyses of controller-pilot communications to detect speedoverrides or other interruptions are incomplete. Interestingly, Center controllers were observed to suspend FIM operations more often than TRACON controllers (9 times). Most of these cases involved perceived separation issues near the meter fixes.

\section{Clearance Phraseology}

Of all the new aspects of the CA-3 operations, clearance phraseology caused the most problems. Study observations noted a number of awkward exchanges, with controllers attempting to resolve issues while consulting the example phraseology lists. The FIM-clearance analysis found 35 of the 128 FIM clearances had problems with phrasing, wrong information, read-back, and/or pilot-input errors. Controllers committed nine of the errors. Eight errors involved the target aircraft; nine involved the target's route. Tables 1 and 2 list the percentage of postsimulation-questionnaire respondents by the ratings they gave FIM-related clearances.

Table 1. Center ratings of FIM-related clearances (1: not at all clear/concise; 7: very clear/concise).

\begin{tabular}{|l|c|c|c|c|c|c|c|}
\hline \multicolumn{1}{|c|}{ Clearance Type } & $\mathbf{1}$ & $\mathbf{2}$ & $\mathbf{3}$ & $\mathbf{4}$ & $\mathbf{5}$ & $\mathbf{6}$ & $\mathbf{7}$ \\
\hline FIM Clearance & $25 \%$ & & & $25 \%$ & & $25 \%$ & $25 \%$ \\
\hline FIM-Engagement Reporting & $25 \%$ & $25 \%$ & & $25 \%$ & & & $25 \%$ \\
\hline Unable-FIM Reporting & & & $25 \%$ & $25 \%$ & $25 \%$ & & $25 \%$ \\
\hline FIM-Status Reporting & & $25 \%$ & $50 \%$ & & $25 \%$ & & \\
\hline FIM Suspension & $25 \%$ & & & $50 \%$ & $25 \%$ & & \\
\hline FIM Resumption & & $25 \%$ & & $50 \%$ & & $25 \%$ & \\
\hline
\end{tabular}

Table 2. TRACON ratings of FIM-related clearances (1: not at all clear/concise; 7: very clear/concise).

\begin{tabular}{|l|c|c|c|c|c|c|c|}
\hline \multicolumn{1}{|c|}{ Clearance Type } & $\mathbf{1}$ & $\mathbf{2}$ & $\mathbf{3}$ & $\mathbf{4}$ & $\mathbf{5}$ & $\mathbf{6}$ & $\mathbf{7}$ \\
\hline FIM Clearance & & $100 \%$ & & & & & \\
\hline FIM-Engagement Reporting & & & $20 \%$ & & $20 \%$ & & $60 \%$ \\
\hline Unable-FIM Reporting & & & & & $40 \%$ & $20 \%$ & $40 \%$ \\
\hline FIM-Status Reporting & & & & & $25 \%$ & $25 \%$ & $50 \%$ \\
\hline FIM Suspension & & & & & $33 \%$ & & $67 \%$ \\
\hline FIM Resumption & & & $25 \%$ & & $25 \%$ & $25 \%$ & $25 \%$ \\
\hline
\end{tabular}

\section{Controller Tools}

In general, the Center controllers were most positive about the tools available to them. Final controllers found the CMS tools least useful, which is line with earlier research indicating Final controllers are most concerned about relative spacing between aircraft. ${ }^{5}$ Tool assessments included the FIM-status designators and spacing cones, in addition to the CMS timelines, early/late indicators, slot markers and speed advisories. Figure 20 shows the TRACON controllers' ratings of how helpful these tools were plotted against their confidence in the information they provided.

The FIM-status designators were highly rated along both dimensions. Controllers toggled them 372 times to monitor the status of the ASTORs that received FIM clearances. On average, the controllers set the status designators approximately 3 times per ASTOR. The status designators were not always easy to set; when and how to set them was a frequent discussion topic, particularly among the Center controllers. The maximum number of recorded changes to an aircraft's FIM status was 10 . 
Final controllers valued the spacing cones, while the slot markers were rated highest among the CMS tools in line with prior research. ${ }^{5}$ The timelines were also highly rated; this could in part be due to the schedule-adjustment functionality they provided. Feeder controllers performed schedule swaps during four simulation trials to avoid having to speed up aircraft that had become late. Again in this study, speed advisories received the lowest ratings. Controllers generally thought winds could affect the usability of the tools, particularly very strong winds.

The Tools conditions with FIM operations also produced a notable effect on the time controllers reported spending on voice communications. Final controllers reported communicating more on average in the Baseline conditions than in the Tools conditions, while both Center and Feeder controllers reported communicating slightly more in the Tools conditions on average.

\section{Workload}

Workload received 'low' ratings throughout the study. While average post-trialquestionnaire responses for 'time pressure' and 'frustration' were below 3 on a scale of 1 to 7 , these ratings were significantly higher in Tools conditions with FIM operations ( $p<0.04$ ). Also, during the trials, a chime signaled controllers to rate their current workload. Final controllers gave consistently low real-timeworkload ratings; the Center-controller and Feeder-controller ratings show a trend similar to the throughput measurements (Fig. 21 and Fig. 22).

\section{Safety and Acceptability}

Controllers were also asked to evaluate the Baseline and Tools conditions using the Controller Acceptance Rating Scale (CARS). ${ }^{11}$ All participants were very positive about the potential of the concept, with no 'unsafe/uncontrollable' ratings. The mode of the responses for each condition was 9 ("Negligible deficiencies. System is acceptable..."); the minimum was 7 ("Minor but annoying deficiencies...") and the maximum 10 ("Deficiencies are rare..."). Center controllers were more positive about the Baseline conditions, while TRACON controllers were more positive about the Tools conditions.

\section{Training}

Controllers gave the training high marks.

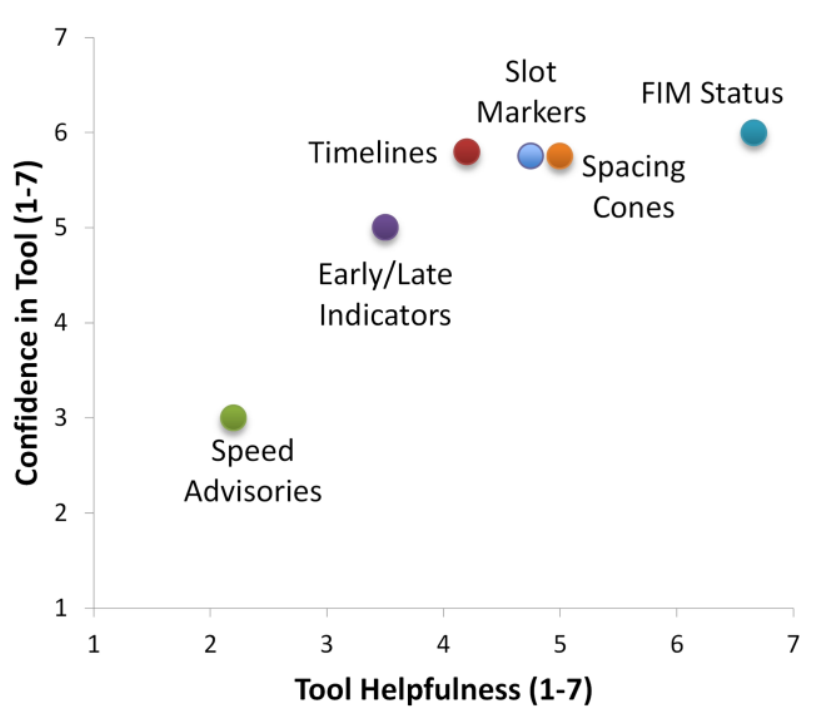

Figure 20. TRACON controller ratings of CA-3 tools.

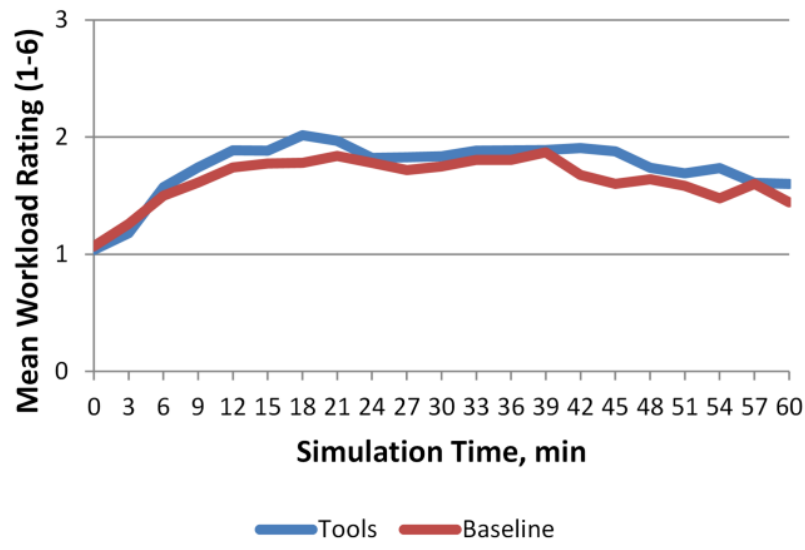

Figure 21. Center controller real-time workload ratings.

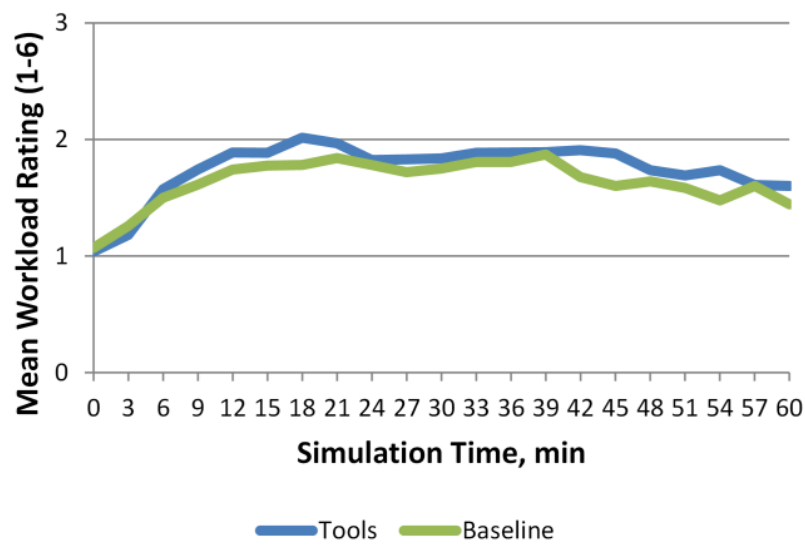

Figure 22. Feeder controller real-time workload ratings.

14

American Institute of Aeronautics and Astronautics 
Average overall ratings for training on the AOL voice-communications system and MACS stations were above 6 on a seven-point scale (1: not very effective; 4: somewhat effective; 7: very effective). Average ratings for the concept introduction, CMS tools, and procedures and phraseology were 5.9, 6, and 5.7, respectively. Once training commenced in the AOL, participants focused on their particular specializations; perhaps due to this, suggestions for improving training focused on the topic of what other actors in the system do. The principal suggestion was to better explain how pilots conduct the FIM operations in the cockpit, perhaps even supplying a 'cheat sheet' on this topic. One TRACON controller felt that, in addition, it would be advantageous to observe how Center controllers handle particular situations. While controllers and pilots clearly became more familiar with the system as CA-3 progressed, enough 'new' situations arose during the course of the study that the results remained fairly consistent, with no overt learning effects.

\section{Summary}

The CA-3 simulation built on integration work conducted for the CA-1 and CA-2 studies to provide a comprehensive view of ATD-1 operations using the Version 1 IM-TAPSS laboratory prototype. Controllers with considerable experience in the study airspace, but who were novices in ATD-1 operations, found the training, tools, procedures, and phraseology usable and acceptable without increasing workload or compromising safety. Center controllers were able to precondiction flows such that aircraft largely conducted OPDs in the TRACON, and FIM operations yielded a small but noticeable increase in inter-arrival precision. Controllers need more practice with the system, particularly in applying the proposed clearance phaseology and maintaining awareness of FIM status.

\section{Conclusion}

The initial series of AOL ground-side-focused simulations for ATD-1 provided an important first look at schedule-based operations with mixed-FIM equipage arrival flows. The IM-TAPSS Version 1 laboratory prototype performed admirably, providing a solid foundation for future ATD-1 development efforts. The simulations exercised key aspects of the ATD-1 ConOps: controller tools, procedures, and clearance phraseology for arrival-flow preconditioning and FIM initiation, OPDs with FIM operations for FIM-equipped aircraft, and OPDs with speed control using CMS tools for unequipped aircraft. Controllers familiar with CMS operations and novices alike found the tools usable and the proposed operations acceptable, with no critical safety issues. CA-3 also hinted at possible roles for TRACON traffic managers in determining suitable scheduling buffers and adjusting schedules as particular traffic situations dictate.

The results of the CA-3 simulation frame reasonable expectations of what could be achieved if the current IMTAPSS system were introduced with limited prior controller and pilot exposure. In particular, FIM operations and related clearance phraseology require more training for consistently smooth operations. Controllers and pilots need a better understanding of the others' challenges and means for addressing them, and clear understanding of how to conduct operations so as to maintain the efficacy of a trajectory-prediction-based system, in order to maximize the benefits it can provide.

The principal ATD-1 benefits mechanisms-well-preconditioned flows to the TRACON, FIM for increased inter-arrival precision, and controller tools to enable speed control of unequipped aircraft on OPDs-depend on each other for success. CA-3 illustrated some beneficial trends, especially in the area of arrival-flow preconditioning using the TMA-TM; arrival flows in CA-3 were consistently better than in the CA-1 and CA-2 studies. FIM operations and TRACON controller tools require not simply controller acceptance, but consistent understanding and application to yield notable incremental benefits. Controllers who conduct ATD-1 operations on a daily basis will likely adapt their work patterns more than the CA-3 controllers. Controllers will be vigilant for disturbances due to factors such as winds and pilotage that can cause an arrival flow to deteriorate. Final controllers, in particular, will judiciously apply speed control in the final-approach region to limit schedule-conformance variability at the FAF and ensure safe spacing at the runway threshold; this in turn maximizes the advantages of FIM operations. Development work is currently underway on IM-TAPSS Version 2, and researchers are preparing to address these issues in upcoming simulations.

\section{Acknowledgments}

This research was conducted as part of the ATD-1 subproject of the NASA Aviation Systems Program, John Robinson III, Technical Lead. The authors wish to thank the many ATD-1 researchers, pilot trainers, and support personnel at NASA Ames and NASA Langley who helped with this research. 


\section{References}

1 ___FAA’s NextGen Implementation Plan,” Federal Aviation Administration, Washington, D.C., March 2011.

${ }^{2}$ Prevot, T., Baxley, B., Callantine, T., Johnson, W., Quon, L., Robinson, J., and Swenson, H., "NASA's ATM Technology Demonstration-1: Transitioning Fuel Efficient, High Throughput Arrival Operations from Simulation to Reality," Proceedings of the International Conference on Human-Computer Interaction in Aerospace (HCI-Aero 2012), Brussels, September 2012.

${ }^{3}$ Robinson III, J. E., “Air Traffic Management Technology Demonstration-1 (ATD-1): Interval Management-Terminal Area Precision Scheduling System Demonstration Description and Plan,” Version 2.5, NASA Ames Research Center, 6 October 2011.

${ }^{4}$ Swenson, H., Thipphavong, J., Sadovsky, A., Chen, L., Sullivan, C., and Martin, L., "Design and Evaluation of the Terminal Area Precision Scheduling and Spacing System," Proceedings of the $9^{\text {th }}$ USA/Europe Air Traffic Management Research and Development Seminar (ATM2011), Berlin, June 2011.

${ }^{5}$ Kupfer, M., Callantine, T., Martin, L., Mercer, J., and Palmer, E., "Controller Support Tools for Schedule-Based Terminal Operations," Proceedings of the $9^{\text {th }}$ USA/Europe Air Traffic Management Research and Development Seminar (ATM2011), Berlin, June 2011.

${ }^{6}$ Murdoch, J., Barmore, B., Baxley, B., Abbott, T., and Capron, W., "Evaluation of an Airborne Spacing Concept to Support Continuous Descent Arrival Operations," Proceedings of the $8^{\text {th }}$ USA/Europe Air Traffic Management Research and Development Seminar (ATM2009), Napa, CA, June 2009.

${ }^{7}$ Baxley, B., Johnson, W., Swenson, H., Robinson, J., Prevot, T., Callantine, T., Scardina, J., and Greene, M., "Air Traffic Management Technology Demonstration-1 Concept of Operations (ATD-1 ConOps)," NASA Technical Memorandum 2012217585, NASA Langley Research Center, Hampton, VA, July 2012.

${ }^{8}$ Baxley, B., Swenson, H., Prevot, T., and Callantine, T., "NASA's ATM Technology Demonstration-1: Integrated Concept of Arrival Operations," Proceedings of the 31 ${ }^{\text {st }}$ Digital Avionics Systems Conference, Williamsburg, VA, October 2012.

${ }^{9}$ Prevot, T., Lee, P., Callantine, T., Mercer, J., Homola, J., Smith, N., and Palmer, E., "Human-in-the-Loop Simulation of NextGen Concepts in the Airspace Operations Laboratory," Proceedings of the AIAA Modeling and Simulation Technologies Conference, Toronto, Ontario, AIAA-2010-7609, 2010.

${ }^{10}$ Cabrall, C., Callantine, T., Kupfer, M., Martin, L., and Mercer, J., "Controller-Managed Spacing Within Mixed-Equipage Arrival Operations Involving Flight Deck Interval Management," Proceedings of the $1^{\text {st }}$ International Conference on Human Factors in Transportation (in AHFE 2012), San Francisco, July 2012.

${ }^{11}$ Lee, K., Kerns, K., Bone, R., and Nickelson, M., "Development and Validation of the Controller Acceptance Rating Scale (CARS): Results of Empirical Research," Proceedings of the $4^{\text {th }}$ USA/Europe Air Traffic Management Research and Development Seminar (ATM2001), Santa Fe, NM, December 2001. 